\title{
Progesterone decreases the extent of ovarian damage caused by cisplatin in an experimental rat model
}

\author{
Saim Ozdamar ${ }^{1, E, F}$, Mine Islimye Taskin ${ }^{2, A, F}$, Gozde Ozge Onder ${ }^{1, B}$, Emin Kaymak ${ }^{1, B}$, Munevver Baran ${ }^{3, C}$, Arzu Yay ${ }^{1, A-F}$ \\ ${ }^{1}$ Department of Histology and Embryology, Medicine Faculty, Erciyes University, Kayseri, Turkey \\ ${ }^{2}$ Department of Obstetrics and Gynecology, Medicine Faculty, University of Balikesir, Turkey \\ ${ }^{3}$ Department of Pharmaceutical Basic Science, Faculty of Pharmacy, Erciyes University, Kayseri, Turkey \\ A - research concept and design; $\mathrm{B}$ - collection and/or assembly of data; $\mathrm{C}$ - data analysis and interpretation; \\ $\mathrm{D}$ - writing the article; $\mathrm{E}$ - critical revision of the article; $\mathrm{F}$ - final approval of the article
}

\section{Address for correspondence \\ Arzu Yay}

E-mail: arzu.yay38@gmail.com

Funding sources

None declared

Conflict of interest

None declared

Received on November 18, 2016

Reviewed on August 1, 2017

Accepted on September 6, 2017

Published online on August 1, 2018

Cite as

Ozdamar S, Islimye Taskin M, Ozge Onder G, Kaymak E,

Baran M, Yay A. Progesterone decreases the extent of ovarian damage caused by cisplatin in an experimental rat model. Adv Clin Exp Med. 2019;28(1):25-33. doi:10.17219/acem/76858

DOI

10.17219/acem/76858

\section{Copyright}

Copyright by Author(s)

This is an article distributed under the terms of the

Creative Commons Attribution Non-Commercial License

(http://creativecommons.org/licenses/by-nc-nd/4.0/)

\begin{abstract}
Background. Apart from the role of progesterone in reproductive physiology, the protective role of exogenously administered progesterone was observed in various injuries, such as neurologic defects and acute kidney injury.
\end{abstract}

Objectives. The aim of the present study was to investigate the effects of progesterone therapy on the immunoexpression of anti-Müllerian hormone (AMH) and the number of apoptotic cells in ovarian damage induced with cisplatin, a chemotherapeutic agent, in an experimental rat model.

Material and methods. Forty rats were randomly divided into 4 groups; the control group (the saline group), the cisplatin-treated group (rats were injected with $5 \mathrm{mg} / \mathrm{kg} /$ week cisplatin intraperitoneally (i.p.)), the cisplatin + progesterone-treated group (the rats were pretreated with $8 \mathrm{mg} / \mathrm{kg}$ progesterone intramuscularly (i.m.) (8 mg/kg) before they were injected with $5 \mathrm{mg} / \mathrm{kg} /$ week cisplatin i.p.), and the progesteronetreated group (the rats were treated with $8 \mathrm{mg} / \mathrm{kg}$ progesterone i.m.). The ovaries were removed from the rats in all groups 5 days after the final injection of cisplatin.

Results. Histopathologic examination and follicle counting were performed. The immunoreactivity intensity of AMH and apoptosis were compared. Histological analysis of the ovaries treated with cisplatin showed ovarian damage. Immunohistochemical analysis showed that the immunoreactivity intensity of AMH, a biomarker that discriminates the degree of ovarian damage, was lower in the cisplatin-treated groups than in other groups. Terminal deoxynucleotide transferase-mediated 20-deoxyuridine 50-triphosphate nick endlabeling (TUNEL) assays showed that the increase in the number of apoptotic cells was statistically significant in the cisplatin-treated group compared to the control group $(p<0.05)$. Progesterone administration with cisplatin resulted in decreases in TUNEL-positive cells. The decrease in the number of apoptotic cells was statistically significant in the cisplatin + progesterone-treated group compared to the control group ( $p<0.001)$.

Conclusions. Our results showed that using progesterone as an adjuvant agent against ovarian damage in patients undergoing cancer chemotherapy with cisplatin is beneficial.

Key words: apoptosis, cisplatin, anti-Müllerian hormone, ovarian damage, progesterone 


\section{Introduction}

Chemotherapy and radiotherapy are the most common modalities of cancer treatment and they have substantially increased the survival period and quality of life in patients with cancer. One of the most important chemotherapeutic agents is cisplatin (cis-diamminedichloroplatinum(II) CDDP), which is currently used in the treatment of a wide range of human tumors, and particularly solid tumors occurring in reproductive structures, like the testes and ovaries. ${ }^{1}$ Unfortunately, chemotherapy treatment strategies give rise to other problems, especially in the reproductive age group, where prolonged chemotherapy exposes a large proportion of these patients to complications that can include infertility, ovarian dysfunction and increased follicular apoptosis. ${ }^{2}$ The use of cisplatin may, therefore, lead to side effects, such as ovarian failure, which limits proper administration of this anticancer drug. ${ }^{3}$ However, the mechanisms that give rise to cisplatin side effects are not clearly understood. For this reason, experimental studies have been initiated to explore methods for preventing reproductive damage in patients who must undergo extensive chemotherapy. ${ }^{4}$ The current literature indicates that the use of antioxidants might be helpful in preventing infertility caused by cisplatin-induced ovarian damage. ${ }^{5}$

Progesterone, a steroid hormone, is necessary for the retention of reproductive functions, such as ovulation and implantation, in females and it organizes the reproductive system. Besides this role in reproductive physiology, progesterone administration is reported to have a protective function against damage occurring during cerebral ischemia reperfusion and due to neurologic defects. ${ }^{6}$ Progesterone is well-documented to protect against injuries of various organs, including the kidney. ${ }^{7}$ For example, exogenously administered progesterone has a protective effect against acute kidney injury in rats subjected to ischemia reperfusion. ${ }^{8}$ Therefore, progesterone may have a similar protective effect in the ovary, and particularly on the ovarian reserve, during chemotherapy.

The status of the ovarian reserve is commonly monitored by anti-Müllerian hormone (AMH). ${ }^{9}$ Also known as Müllerian inhibiting substance, $\mathrm{AMH}$ is a $140 \mathrm{kDa}$ polypeptide responsible for multiple reproductive functions. Yeh et al. recently showed that AMH is a better indicator of the ovarian reserve during the aging process than certain other ovarian hormones, as it is a necessary hormone for the growing follicular pool. ${ }^{10}$ Recent AMH studies suggest that chemotherapeutic drugs might cause ovarian damage and play a role in the failure of the ovarian reserve. ${ }^{3}$ However, in humans, few approaches are available that can protect patient's fertility and ovaries while applying chemotherapy. ${ }^{11}$

For these reasons, we hypothesized that progesterone might exert a protective effect on cisplatin-induced ovarian damage. The protective effects of progesterone have been previously studied, but no animal model has yet been developed for the study of the potential protective effect of progesterone on cisplatin-induced ovarian damage. Therefore, this study aimed to establish a rat model for the assessment of the effects of progesterone on cisplatin-induced ovarian damage via histopathological evaluation. The overall goal of the present study was to investigate whether progesterone can protect the ovaries of female rats against follicular damage during cisplatin chemotherapy.

\section{Material and methods}

\section{Animals and drug administration}

All experimental protocols were conducted in accordance with the institutional guidelines for the experimentation on animals at Erciyes University, Faculty of Medicine (Kayseri, Turkey), and the study was approved by the research ethics board of Erciyes University. For this study, 36 healthy female adult Wistar rats were obtained from Hakan Cetinsaya Experimental and Clinical Research Center of Erciyes University. All the animals were housed in plastic cages placed in a well-ventilated rat house, given ad libitum access to food and water, and subjected to a natural photoperiod of a 12-hour light/12-hour dark cycle. The animals were treated in accordance with the Guidelines for Animal Experimentation of Jichi Medical University (Shimotsuke, Japan), based on the National Institutes of Health Guidelines for the Care and Use of Laboratory Animals.

The rats were randomly assigned to 4 groups: the saline group $(n=8)$, the cisplatin-treated group $(n=10)$, the progesterone + cisplatin-treated group $(n=10)$, and the progesterone-treated group $(n=8)$. The rats in the cisplatin group were administered a single intraperitoneal (i.p.) injection of cisplatin (5 mg/kg; Eczacibasi, Istanbul, Turkey). The rats in the control group were similarly administered the same volume of $0.9 \%$ sodium chloride $(\mathrm{NaCL})$. Rats subjected to progesterone treatment were administered progesterone $(8 \mathrm{mg} / \mathrm{kg}$; Kocak Farma, Istanbul, Turkey) intramuscularly (i.m.) 30 min before the injection of cisplatin in the progesterone + cisplatin-treated group, or saline in the progesterone-treated group. All injections were repeated 1 week later. Five days after the $2^{\text {nd }}$ injection, the rats were sacrificed by decapitation under i.p. ketamine $(50 \mathrm{mg} / \mathrm{kg})$ xylazine $(10 \mathrm{mg} / \mathrm{kg})$ anesthesia. After decapitation, the right and left ovaries were immediately excised.

\section{Histological analysis}

A morphological overview of the structure of the ovarian tissue was obtained using routine histological methods. Specimens were fixed in $10 \%$ formalin solution for $24-48 \mathrm{~h}$, embedded in paraffin wax and cut into $5-\mu \mathrm{m}$ thick sections. The slides were cleared in xylene, dehydrated in an ascending alcohol chain, and stained with 
hematoxylin-eosin $(\mathrm{H} \& \mathrm{E})$ for the evaluation of the tissue morphology and structure. Photographs were taken with a photomicroscope (Olympus BX51; Olympus, Tokyo, Japan) and analyzed.

The evaluation scale suggested by Li et al. was applied, with some modifications, for the histopathological evaluation of the ovarian tissues. ${ }^{12}$ The histological sections were examined for the presence of vascular congestion, hemorrhage, follicular degeneration, leukocyte infiltration, and interstitial edema. The histological changes were scored from 0 to 3 according to the histological findings, where 0 represents no pathological findings, and 1, 2 and 3 represent pathological findings in $<33 \%, 33-66 \%$ and $>66 \%$ of the ovary, respectively. The scores for each parameter were calculated and the total scores were obtained.

Follicle counting and classification were performed on every $12^{\text {th }}$ section stained with H\&E. All measurements were carried out only in follicles with an obvious nucleus, using ImageJ software (ImageJ, Bethesda, USA). The assessment of follicular quality was based on the cellular density and the integrity of the basement membrane and the oocyte. The follicles were classified as morphologically normal and only normal follicles were quantified according to these criteria. They were then classified in the histological preparations, based on stage, into primordial, primary, secondary, or antral follicles. Briefly, primordial follicles were defined as an oocyte surrounded by 1 single epithelial cell layer; primary follicles were characterized by a single cell layer with cubic or high-prismatic cells; secondary follicles consisted of multiple layers of granulosa cells; and Graafian follicles were defined as those with antrum folliculi, stratum granulosum and cumulus oophorus.

\section{Immunohistochemistry}

The immunoexpression of AMH within the follicles of the ovaries was detected using the avidin-biotin-peroxidase method (ImmunoCruz ${ }^{\text {TM }}$ Staining System, Sc:2053; Santa Cruz Biotechnology Inc., Santa Cruz, USA), following the manufacturer's recommendations. Briefly, serial 5 - $\mu \mathrm{m}$ thick paraffin-embedded sections were deparaffinized, rehydrated in graded alcohol and incubated for $5 \mathrm{~min}$ in phosphate buffered saline (PBS) at room temperature. Antigen retrieval was carried out by microwave treatment in $0.01 \mathrm{M}$ sodium citrate buffer ( $\mathrm{pH} 6.0$ ) at $95^{\circ} \mathrm{C}$ for $15 \mathrm{~min}$. The specimens were washed several times with PBS and endogenous peroxidase activity was inhibited with $3 \%$ hydrogen peroxide in methanol for $10 \mathrm{~min}$. The specimens were incubated with a serum-blocking agent to block nonspecific staining. The histological sections were then incubated overnight at $4{ }^{\circ} \mathrm{C}$ with a $1 / 50$ dilution of goat polyclonal anti-AMH antibody (MIS (C-20): sc-6886; Santa Cruz Biotechnology Inc.). For immunostaining assays, primary antibodies were omitted as a negative control. After washing with PBS, the sections were incubated for $15 \mathrm{~min}$ with the biotinylated secondary antibodies. The sections were visualized by treatment for 3-5 min at room temperature with 3,3-P-diaminobenzidine tetrahydrochloride (DAB) as a chromogen and then lightly counterstained with hematoxylin. Images were taken using an immunofluorescence microscope (Olympus BX51; Olympus). We selected primordial, primary, preantral, secondary, and mature or Graafian follicles in sections from each experimental group at the same magnification $(\times 40)$. The mean immunoreactivity intensity for each follicle in an ovarian section was measured using ImageJ software (ImageJ).

\section{TUNEL staining}

In situ detection of apoptosis was performed in the sections by terminal deoxynucleotide transferase-mediated 20-deoxyuridine 50-triphosphate nick end-labeling (TUNEL), using an In situ Cell Death Detection Kit (Chemicon, Temecula, USA), according to the manufacturer's instructions. Briefly, paraffin sections $(5-\mu \mathrm{m})$ from the ovarian tissues were deparaffinized, rehydrated and washed twice in PBS for $5 \mathrm{~min}$. The TUNEL reaction mixture was added to each slide and incubated in a humidified atmosphere for $60 \mathrm{~min}$ at $37^{\circ} \mathrm{C}$ in the dark. After washing with PBS, the sections were then incubated with a converter reagent for $30 \mathrm{~min}$. Color development for the localization of cells containing labeled DNA strand breaks was performed by incubating the slides with Fast Red Substrate System (F4648; Sigma-Aldrich Chemie GmbH, Taufkirchen, Germany) for $5 \mathrm{~min}$. Finally, the association with the number of TUNEL-positive cells in the rats from the experimental groups was evaluated by identifying apoptotic cells by morphology. At least 3 randomly chosen fields in each slide were counted at the original $\times 20$ magnification. All slides were examined and photographed using an Olympus BX51 microscope (Olympus). The numbers of apoptotic cells were determined by carefully counting the TUNELpositive cells, using ImageJ software (ImageJ) at the same magnification. ${ }^{2}$

\section{Statistical analysis}

Statistical significance was evaluated using GraphPad Prism v. 5.01 (Graph Pad Software Inc., San Diego, USA). The one-way analysis of variance (ANOVA) was followed by an appropriate post hoc comparison (depending on the given Gaussian distribution) and all the data was presented as the mean of normalized data \pm standard error of the mean (SEM). The results were considered significant if the $\mathrm{p}$-value was $<0.05$.

\section{Results}

The histological sections stained with $H \& E$ revealed that the control and progesterone-treated groups had normal ovarian architecture with no considerable pathologic 

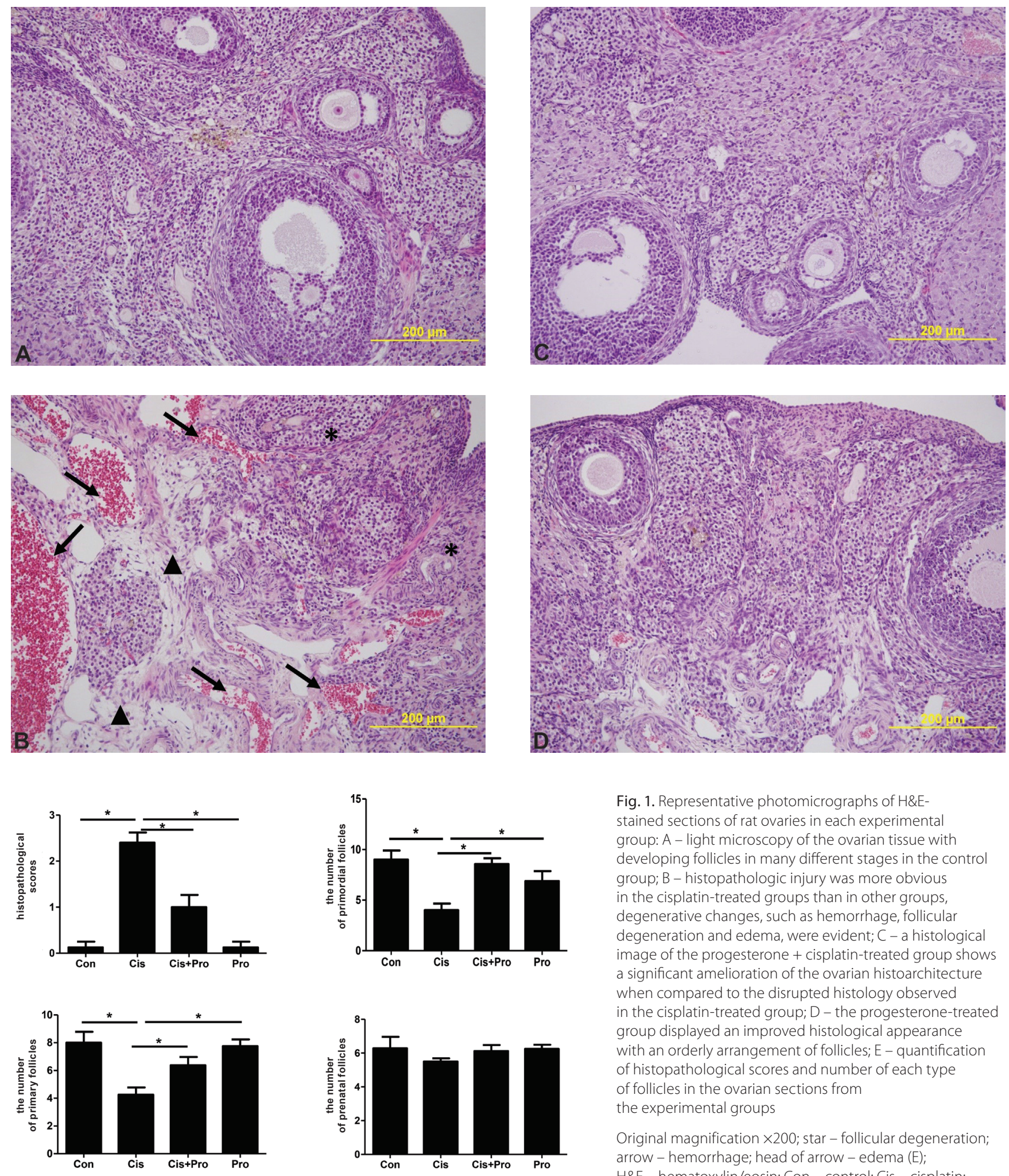

Fig. 1. Representative photomicrographs of H\&Estained sections of rat ovaries in each experimental group: A - light microscopy of the ovarian tissue with developing follicles in many different stages in the control group; B - histopathologic injury was more obvious in the cisplatin-treated groups than in other groups, degenerative changes, such as hemorrhage, follicular degeneration and edema, were evident; $\mathrm{C}$ - a histological image of the progesterone + cisplatin-treated group shows a significant amelioration of the ovarian histoarchitecture when compared to the disrupted histology observed in the cisplatin-treated group; D - the progesterone-treated group displayed an improved histological appearance with an orderly arrangement of follicles; E - quantification of histopathological scores and number of each type of follicles in the ovarian sections from the experimental groups

Original magnification $\times 200$; star - follicular degeneration; arrow - hemorrhage; head of arrow - edema (E): H\&E - hematoxylin/eosin; Con - control; Cis - cisplatin; Pro - progesterone; ${ }^{*} \mathrm{p}<0.05$.
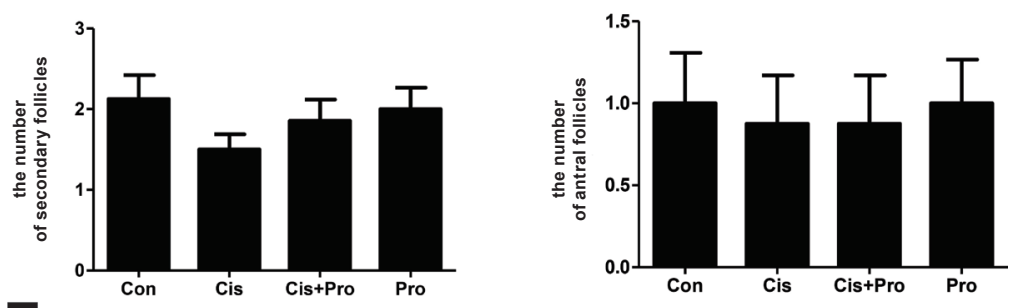

E 
alteration (Fig. 1). Ovarian follicles in various stages of development and with normal appearance were observed in the ovarian cortex, and the cuboid germinal or follicular cells around the oocyte were examined. The cisplatininjected group showed the absence of Graafian follicles and the presence of the corpus luteum (CL), whereas the sections taken from the control ovarian tissues exhibited the presence of follicles at different stages of maturation. Cisplatin treatment also resulted in microscopy findings of hemorrhage and vascular congestion, as well as follicular degeneration and edema in the ovarian tissue when compared to the control group. The group that was administered cisplatin + progesterone showed more moderate, but morphologically similar, histological findings of degeneration, hemorrhage and edema. Overall, progesterone markedly decreased cisplatin-induced ovarian damage.

For follicle counting at different stages in each experimental group, unhealthy follicles were distinguished from healthy follicles by nuclear pyknosis and the disappearance of granulosa cells, whereas healthy follicles were classified considering their different developmental stages. The control group showed a number of follicles at various stages, whereas significantly fewer primordial, secondary and Graafian follicles were found in the cisplatin group than in the control group ( $\mathrm{p}<0.05)$, indicating substantial damage to the quality of the follicle in the cisplatin group. Primordial and primary follicle counts were higher in the control group than in the cisplatin group, and this difference was statistically significant $(\mathrm{p}<0.05)$. Primordial and primary follicle counts were similar in the cisplatin + progesterone-treated group and the cisplatin group ( $\mathrm{p}<0.05$ ). The numbers of preantral, secondary and Graafian follicles did not differ among the groups. Preantral, secondary and Graafian follicle counts were lower in the cisplatin + progesterone-treated group than in the cisplatin-treated group. No statistically significant differences were seen between the control and progesterone groups regarding the follicle numbers (Fig. 1E). All histological scores are listed in Table 1. The histopathological differences were marked as follows: 0 - normal; 1 - moderate (moderate vascular congestion, edema, no hemorrhage, and no follicular degeneration); 2 - moderate (moderate vascular congestion, edema, no hemorrhage, and no follicular degeneration); and 3 - severe (severe vascular congestion, edema, hemorrhage, follicular degeneration, and leukocyte infiltration).

Table 1. Histopathological grading of rat ovary lesions in the treatment groups

\begin{tabular}{|l|c|c|c|c|}
\hline \multirow{2}{*}{\multicolumn{1}{c|}{ Groups }} & \multicolumn{5}{c|}{ Histopathological scores } \\
\cline { 2 - 5 } & 0 & 1 & 2 & 3 \\
\hline Control & 8 & - & - & - \\
\hline Cisplatin & - & 1 & 4 & 5 \\
\hline Cisplatin + progesterone & 2 & 4 & 2 & 2 \\
\hline Progesterone & 7 & 1 & - & - \\
\hline
\end{tabular}

The influence of progesterone on AMH immunoreactivity intensity in the ovaries of the rats in all experimental groups is shown in Fig. 2. Anti-Müllerian hormone immunohistochemistry was analyzed by follicle class. No AMH was detected in atretic follicles, theca cells and the oocytes of follicles in the ovarium. Anti-Müllerian hormone was primarily expressed in granulosa cells of primary, preantral and small antral follicles. Anti-Müllerian hormone immunoreactivity intensity progressively decreased in the subsequent phases of follicle development in the control and progesterone-treated groups, whereas the immunoreactivity intensity of AMH was consistently lower in the ovaries of the cisplatin-treated group. The immunoreactivity intensity of $\mathrm{AMH}$ was lower, especially in primordial and primary follicles at early development stages, in the cisplatin-administered group than in the control group, but the differences were not statistically significant. The immunoreactivity intensity of AMH did not differ between secondary and tertiary follicles (Fig. 2E).

The TUNEL evaluations for DNA fragmentation in cell nuclei in each group are shown in Fig. 3. The control group showed no increases in the numbers of apoptotic cells in follicles at different stages. By contrast, the numbers of TUNEL-positive cells were significantly higher in the cisplatin-treated groups than in the control group $(\mathrm{p}<0.001)$. The cisplatin group showed badly damaged follicle structures, resulting in a significant rise in the number of TUNEL-positive cells. Markedly lower numbers of TUNEL-positive cells were seen in follicles of the progesterone-treated groups than in the cisplatin-treated group ( $\mathrm{p}<0.001$ ). Thus, the induction of large numbers of TUNEL-positive cells in the ovarian tissue by cisplatin treatment was significantly suppressed by pretreatment with progesterone (Fig. 3).

\section{Discussion}

The present study investigated whether progesterone could protect against cisplatin-induced ovarian damage in a rat model. We demonstrated that an injection of progesterone $30 \mathrm{~min}$ prior to cisplatin administration attenuated the cisplatin-induced ovarian injury by reducing cellular apoptosis. Progesterone also positively modulated $\mathrm{AMH}$ expression, indicating a possible protection of the ovarian reserve against cisplatin-induced injury. In addition, administration of progesterone led to partial protection. Evaluation of the ovarian reserve following chemotherapy is vital to ensure adequate subsequent fertility.

The targets of the chemotherapeutic drugs might be the granulosa cells in developing follicles, which may affect oocyte maturation and cause follicular destruction, followed by ovarian failure as the result of any damage to these cells. Follicle damage induced by chemotherapy is likely the main cause of infertility. For example, Meirow et al. reported that chemotherapy, in a drug- or dose-addiction, causes 

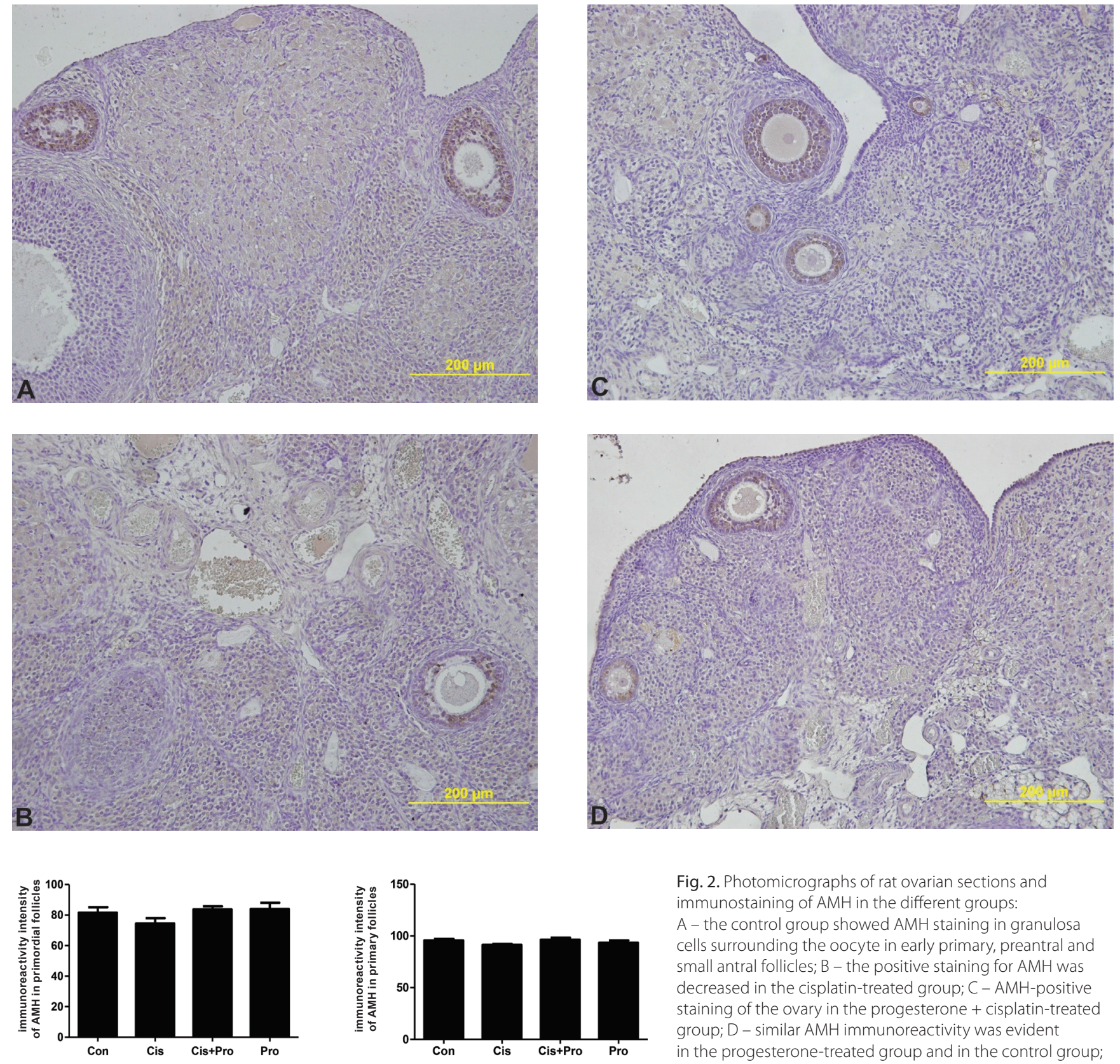

Fig. 2. Photomicrographs of rat ovarian sections and immunostaining of $\mathrm{AMH}$ in the different groups: $\mathrm{A}$ - the control group showed $\mathrm{AMH}$ staining in granulosa cells surrounding the oocyte in early primary, preantral and small antral follicles; $\mathrm{B}$ - the positive staining for $\mathrm{AMH}$ was decreased in the cisplatin-treated group; $\mathrm{C}-\mathrm{AMH}$-positive staining of the ovary in the progesterone + cisplatin-treated group; D - similar AMH immunoreactivity was evident in the progesterone-treated group and in the control group; $\mathrm{E}$ - quantification of AMH immunoreactivity intensity in ovarian sections from the experimental groups
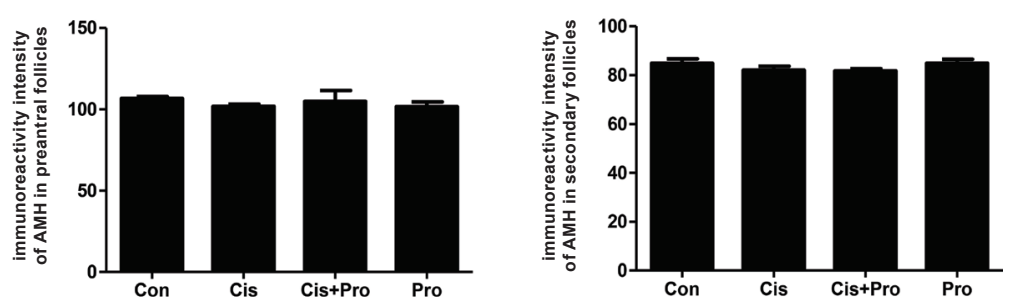

Original magnification $\times 200$; AMH - anti-Müllerian hormone; Con - control; Cis - cisplatin; Pro - progesterone.

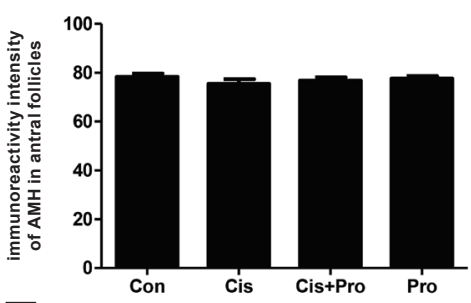

E 

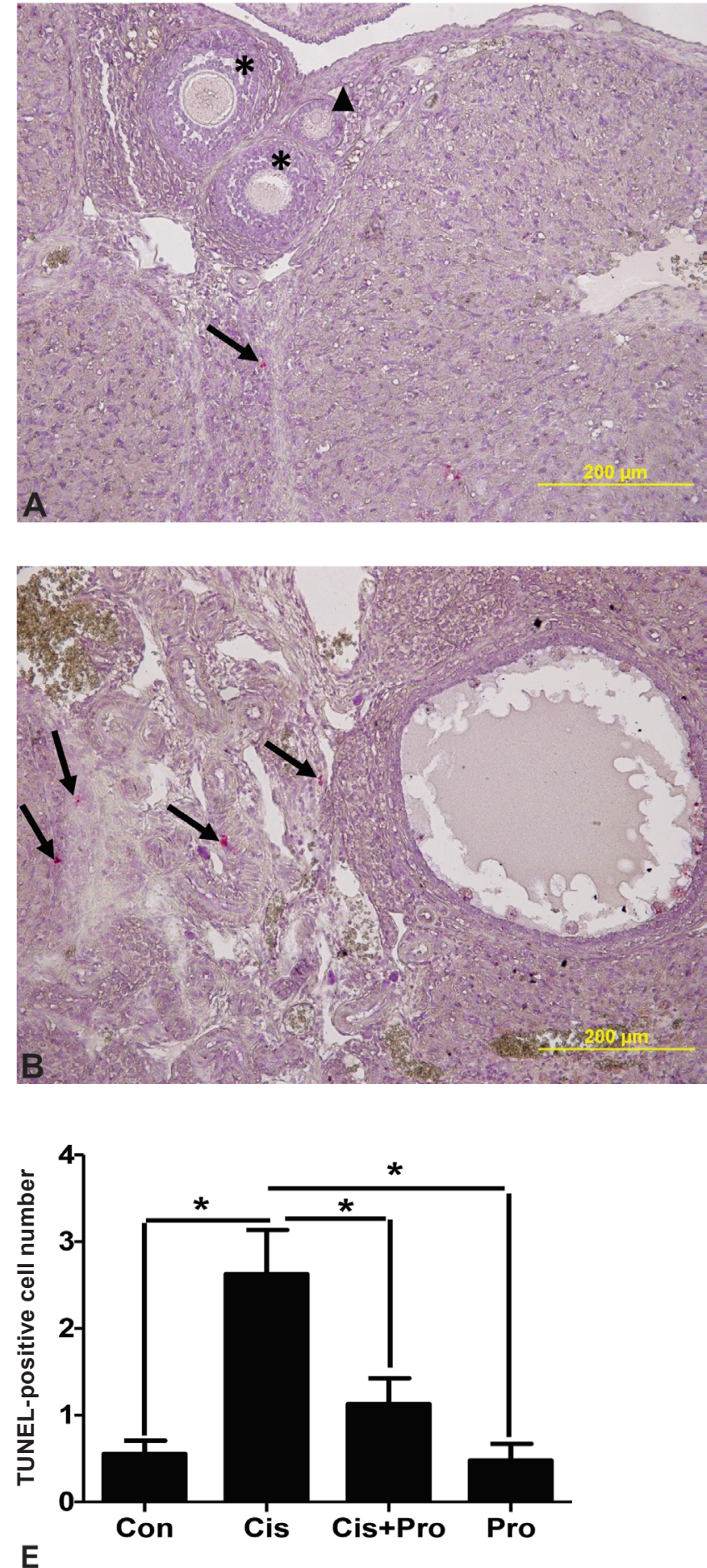

Fig. 3. Progesterone pretreatment inhibits the development of TUNEL-positive cells in the rat ovary due to cisplatin-induced ovarian damage: A - only a few TUNEL-positive cells were evident in the ovaries in the control group; $B$ - the TUNEL-positive cell number increased in the cisplatin-treated group; C - effects of progesterone on TUNEL-positive cells in cisplatin injury in rats, a marked decrease was evident in the number of TUNEL-positive cells when compared with the cisplatin-treated group; D - the progesterone-treated group had also fewer TUNEL-positive cells than were observed in the control group; E - graph showing quantification of the different groups. The number of TUNEL-positive cells was higher in the ovarian tissues of the cisplatin-treated group than in the control group and in other groups

Arrow - TUNEL-positive cell; star - preantral follicle; head of arrow - primary follicle; thick arrow - secondary follicle; TUNEL - terminal deoxynucleotide transferase-mediated 20-deoxyuridine 50-triphosphate nick end-labeling; Con - control; Cis - cisplatin; Pro - progesterone; TUNEL staining $\times 200$; ${ }^{*} p<0.001$.
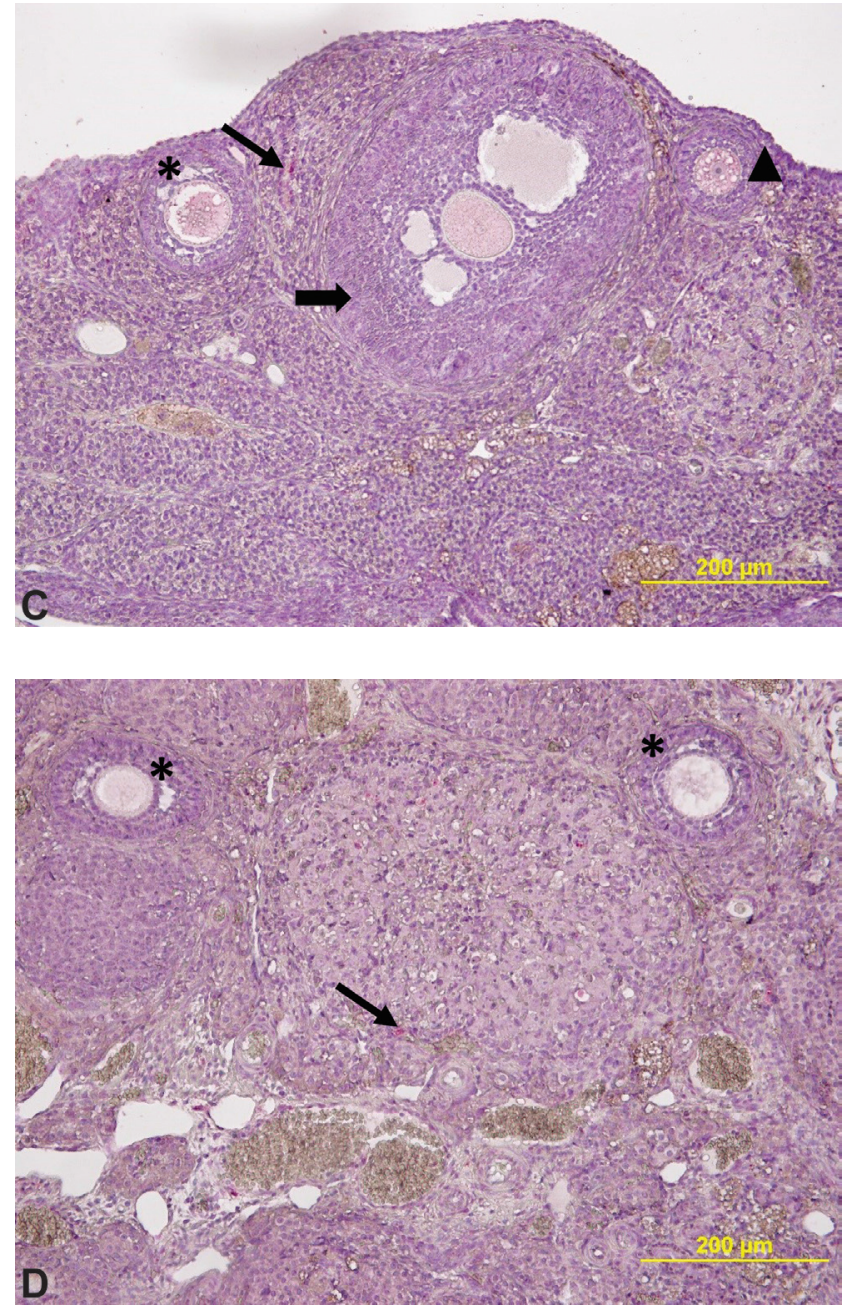

a depletion of the primordial follicle pool. ${ }^{13}$ Treatment with cisplatin causes an increased prevalence of premature ovarian failure in humans. ${ }^{14}$ In rats, cisplatin can cause ovarian damage, decreased immunoreactivity intensity of $\mathrm{AMH}$ and changes in the estrous cycle. ${ }^{15}$

Cisplatin is the commonly preferred anticancer agent for treatment of a wide range of cancer types, ranging from sarcomas and small cell lung cancer to ovarian cancer, cervical cancer and lymphomas. ${ }^{16}$ The antitumor effect of cisplatin depends on the formation within the cell of a platinum complex that participates in DNA cross-linking and apoptosis. ${ }^{17}$ In addition, cisplatin is thought to trigger DNA damage, failure of mitochondrial function and a hindrance in protein synthesis. ${ }^{1,18,19}$ However, these effects on dividing cancer cells may also adversely occur in healthy dividing cells, such as the granulosa cells of follicles, thereby leading to ovarian failure, and ultimately, infertility. ${ }^{12}$

A positive role of progesterone has been indicated in some disorders, such as traumatic brain and spinal cord injury, neuroinflammation, stroke, ischemia, diabetic neuropathy, and neurodegeneration..$^{20,21}$ The granulosa cells and the CL in the ovary produce progesterone, which is necessary in mammals for the establishment and maintenance of pregnancy. ${ }^{22}$ The production of interleukin (IL)- 6 can 
also be inhibited by progesterone, suggesting a probable immunosuppressive and anti-inflammatory role as well. ${ }^{23}$ He et al. showed that progesterone reduces brain edema, ameliorates brain complications with its anti-inflammatory effects and reduces the levels of inflammatory cytokines. ${ }^{24}$ Progesterone also suppresses the production of proinflammatory cytokines and chemokines in monocytes, and appears to play an important anti-inflammatory role. ${ }^{25,26}$ Ghasemi et al. demonstrated a beneficial and dose-dependent effect of progesterone on cisplatininduced nephrotoxicity. ${ }^{7}$ However, the protective effect of progesterone against cisplatin-caused ovarian damage has not been studied extensively.

In the present study, AMH expression was significantly increased in the progesterone + cisplatin-treated group when compared to the cisplatin-treated group, indicating that progesterone can preserve the granulosa cells in growing follicles. No significant increase was observed in $\mathrm{AMH}$ immunoreactivity intensity in the progesterone-treated group when compared to the control group. Our results indicated that pretreatment with progesterone before the administration of cisplatin could have protected the follicle cells from the adverse effects of cisplatin, as indicated by the higher $\mathrm{AMH}$ immunoreactivity intensity in the progesterone + cisplatin-treated group as compared to the cisplatin-treated group. Anti-Müllerian hormone knockout mouse studies have shown that AMH functions as a negative stimulator of follicular maturation and an inhibitor of follicle-stimulating hormone (FSH) sensitivity. ${ }^{27}$

Analyzing the apoptotic mechanism is crucial in explaining the adverse cisplatin effects and the mechanisms underlying the action of possible protective agents against these effects. The ideal preventative or protective agents also must not disturb the antitumor effects of chemotherapeutics. Cisplatin can cause the generation of oxygen-free radicals, which then induce oxidative stress that gives rise to cisplatin-induced tissue damage. ${ }^{28}$ Progesterone used together with chemotherapeutic drugs clearly decreased apoptosis in the present study. Devarajan et al. demonstrated that cisplatin induced both the intrinsic and the extrinsic apoptotic pathways. ${ }^{29}$ Progesterone may decrease apoptosis by decreasing pro-apoptotic enzymes and increasing anti-apoptotic proteins. ${ }^{30}$ In the present study, the antiapoptotic effect of progesterone on cisplatin-induced ovarian damage was confirmed by the reduction in the number of TUNEL-positive apoptotic cells in the progesterone + cisplatin-treated group.

This study provides additional details about the damage caused by cisplatin to the ovary. It also presents methods for determining the protective effect of progesterone against this damage and would appear to be the first report on the effect of progesterone on cisplatin-induced ovarian damage and the immunoreactivity intensity of AMH. Taken together, our results indicate that progesterone partially prevents the damage caused by cisplatin. This finding could be crucial for women cancer patients to maintain their fertility.
In summary, we obtained data from a rat model that support the hypothesis that apoptosis can be regulated by the natural sex steroid progesterone. Additional clinical and immunohistochemical research should be carried out to confirm this conclusion about the ameliorating effect of progesterone on ovarian tissue damage, and to evaluate the efficacy, safety and potential use of progesterone.

\section{References}

1. Wang D, Lippard SJ. Cellular processing of platinum anticancer drugs. Nat Rev Drug Discov. 2005;4(4):307-320.

2. Taskin MI, Yay A, Adali E, Balcioglu E, Inceboz U. Protective effects of sildenafil citrate administration on cisplatin-induced ovarian damage in rats. Gynecol Endocrinol. 2015;31(4):272-277.

3. Yeh J, Kim BS, Liang YJ, Peresie J. Gonadotropin stimulation as a challenge to calibrate cisplatin induced ovarian damage in the female rat. Reprod Toxicol. 2009;28:556-562.

4. Yazici A, Sari ES, Yay A, et al. The protective effect of selenium in cisplatin-related retinotoxicity. Cutan Ocul Toxicol. 2014;33(4):327-332.

5. De Castilhos J, Hermel EE, Rasia-Filho AA, Achaval M. Influence of substitutive ovarian steroids in the nuclear and cell body volumes of neurons in the posterodorsal medial amygdala of adult ovariectomized female rats. Neurosci Lett. 2010;469(1):19-23.

6. Cheng J, Zhang J, Ma X, Su D. Frequency-dependent acceleration of cardiac repolarization by progesterone underlying its cardiac protection against drug-induced proarrhythmic effects in female rabbits. Eur J Pharmacol. 2012;689(1-3):172-178.

7. Ghasemi M, Nematbakhsh M, Pezeshki Z, Soltani N, Moeini M, Talebi A. Nephroprotective effect of estrogen and progesterone combination on cisplatin-induced nephrotoxicity in ovariectomized female rats. Indian J Nephrol. 2016;26(3):167-175.

8. Sandhi J, Singh JP, Kaur T, Ghuman SS, Singh AP. Involvement of progesterone receptors in ascorbic acid-mediated protection against ischemia-reperfusion-induced acute kidney injury. J Surg Res. 2014;187(1):278-288.

9. Themmen AP. Anti-Müllerian hormone: Its role in follicular growth initiation and survival and as an ovarian reserve marker. J Nat/ Cancer Inst Monogr. 2005;34:18-21.

10. Yeh J, Kim BS, Peresie J, Liang YJ, Arroyo A. Serum and ovarian Müllerian inhibiting substance, and their decline in reproductive aging. Fertil Steril. 2007;87(5):1227-1230.

11. Lee SJ, Schover LR, Partridge AH, et al.; American Society of Clinical Oncology. American Society of Clinical Oncology recommendations on fertility preservation in cancer patients. J Clin Oncol. 2006;24(18):2917-2931.

12. Li X, Kang X, Deng Q, Cai J, Wang Z. Combination of a GnRH agonist with an antagonist prevents flare-up effects and protects primordial ovarian follicles in the rat ovary from cisplatin-induced toxicity: A controlled experimental animal study. Reprod Biol Endocrinol. 2013;11:16. doi: 10.1186/1477-7827-11-16

13. Meirow D, Lewis $H$, Nugent D, Epstein M. Subclinical depletion of primordial follicular reserve in mice treated with cyclophosphamide: Clinical importance and proposed accurate investigative tool. Hum Reprod. 1999;14(7):1903-1907.

14. Meirow D. Reproduction post-chemotherapy in young cancer patients. Mol Cell Endocrinol. 2000;169(1-2):123-131.

15. Yucebilgin MS, Terek MC, Ozsaran A, et al. Effect of chemotherapy on primordial follicular reserve of rat: An animal model of premature ovarian failure and infertility. Aust N Z J Obstet Gynaecol. 2004;44(1):6-9.

16. Erken HA, Koç ER, Yazıcı H, et al. Selenium partially prevents cisplatin-induced neurotoxicity: A preliminary study. Neurotoxicology. 2014;42:71-75.

17. Meraner V, Gamper EM, Grahmann A, et al. Monitoring physical and psychosocial symptom trajectories in ovarian cancer patients receiving chemotherapy. BMC Cancer. 2012;12:77. https://doi. org/10.1186/1471-2407-12-77

18. Dasari S, Tchounwou PB. Cisplatin in cancer therapy: Molecular mechanisms of action. Eur J Pharmacol. 2014;740:364-378 
19. Lomeli N, Di K, Czerniawski J, Guzowski JF, Bota DA. Cisplatin-induced mitochondrial dysfunction is associated with impaired cognitive function in rats. Free Radic Biol Med. 2017;102:274-286.

20. Coronel MF, Labombarda F, Roig P, Villar MJ, De Nicola AF, González SL. Progesterone prevents nerve injury-induced allodynia and spinal NMDA receptor upregulation in rats. Pain Med. 2011;12(8):1249-1261.

21. Liu A, Margaill I, Zhang S, et al. Progesterone receptors: A key for neuroprotection in experimental stroke. Endocrinology. 2012;153(8):3747-3757.

22. Graziano V, Check JH, Dietterich C, Choe JK, Yuan W. A comparison of luteal phase support in graduated estradiol/progesterone replacement cycles using intramuscular progesterone alone versus combination with vaginal suppositories on outcome following frozen embryo transfer. Clin Exp Obstet Gynecol. 2005;32(2):93-94.

23. Li S, Yixi S, Feng M, Lü P, Huang H, Zhou J. Progesterone inhibits Tolllike receptor 4-mediated innate immune response in macrophages by suppressing NF-KB activation and enhancing SOCS1 expression. Immunol Lett. 2009;125(2):151-155.

24. He J, Evans CO, Hoffman SW, Oyesiku NM, Stein DG. Progesterone and allopregnanolone reduce inflammatory cytokines after traumatic brain injury. Exp Neurol. 2004;189(2):404-412.
25. Miller L, Hunt JS. Regulation of TNF alpha production in activated mouse macrophages by progesterone. J Immunol. 1998;160: 5098-5104.

26. Hardy DB, Janowski BA, Corey DR, Mendelson CR. Progesterone receptor plays a major anti-inflammatory role in human myometrial cells by antagonism of nuclear factor-kappaB activation of cyclooxygenase 2 expression. Mol Endocrinol. 2006;20(11):2724-2733.

27. Visser JA, Themmen AP. Anti-Müllerian hormone and folliculogenesis. Mol Cell Endocrinol. 2005;234(1-2):81-86.

28. Cepeda V, Fuertes MA, Castilla J, Alonso C, Quevedo C, Pérez JM. Biochemical mechanisms of cisplatin cytotoxicity. Anticancer Agents Med Chem. 2007;7(1):3-18.

29. Devarajan P, Savoca M, Castaneda MP, et al. Cisplatin-induced apoptosis in auditory cells: Role of death receptor and mitochondrial pathways. Hear Res. 2002;174(1-2):45-54.

30. Yao XL, Liu J, Lee E, Ling GS, McCabe JT. Progesterone differentially regulates pro- and antiapoptotic gene expression in cerebral cortex following traumatic brain injury in rats. $J$ Neurotrauma. 2005;22(6):656-668. 